\title{
Chronic obstructive pulmonary disease and long-term mortality in elderly subjects with chronic heart failure
}

\author{
Gianluca Testa $^{1} \cdot$ Francesco Cacciatore $^{2,3} \cdot$ Andrea Bianco $^{4} \cdot$ David Della-Morte $^{5,6} \cdot$ Francesca Mazzella $^{2}$. \\ Gianluigi Galizia $^{7} \cdot$ Gaetano Gargiulo $^{8} \cdot$ Francesco Curcio $^{2} \cdot$ Ilaria Liguori $^{2} \cdot$ Alexandra Sabusco $^{1} \cdot$ Franco Rengo $^{3}$. \\ Domenico Bonaduce $^{2} \cdot$ Pasquale Abete $^{2}$
}

Received: 26 October 2016 / Accepted: 23 December 2016 / Published online: 21 February 2017

(C) Springer International Publishing Switzerland 2017

\begin{abstract}
Background Chronic heart failure (CHF) and chronic obstructive pulmonary disease (COPD) are associated with high rates of mortality in elderly subjects. Concurrent CHF and COPD frequently occur, especially in with advancing age. This study examines long-term mortality in community-dwelling elderly subjects affected by CHF alone, COPD alone, and coexistent CHF and COPD.

Methods The study evaluated 12-years mortality in 1288 subjects stratified for the presence or absence of CHF or COPD alone, and for coexistence of CHF and COPD.

Results Mortality, at 12 year follow-up, was $46.7 \%$ overall, $68.6 \%$ in the presence of CHF alone $(p<0.001)$, $56.9 \%$ in the presence of COPD alone $(p<0.01)$; mortality was $86.2 \%$ where CHF and COPD coexisted $(p<0.001)$ and was significantly higher than in CHF or COPD alone
\end{abstract}

Gianluca Testa

gianluca.testa@unimol.it

1 Department of Medicine and Health Sciences "Vincenzo Tiberio", University of Molise, 86100, Campobasso, Italy

2 Department of Translational Medical Sciences, University of Naples "Federico II", Naples, Italy

3 IRCCS Salvatore Maugeri Foundation, Scientific Institute of Campoli/Telese, Benevento, Italy

4 Department of Cardio-Thoracic and Respiratory Sciences, Second University of Naples, Naples, Italy

5 Department of Systems Medicine, University of Rome Tor Vergata, Rome, Italy

6 IRCCS San Raffaele Pisana, Rome, Italy

7 IRCCS Salvatore Maugeri Foundation, Scientific Institute of Veruno, Novara, Italy

8 Division of Geriatrics, AON SS. Antonio e Biagio e Cesare Arrigo, Alessandria, Italy $(p<0.05)$. Multivariate analysis indicates that CHF (Hazard risk $=1.67,95 \%$ confidence interval 1.15-3.27, $p<0.031$ ) and COPD (Hazard risk $=1.27,95 \%$ confidence interval $=1.08-1.85, p<0.042$ ) were predictive of longterm mortality. When CHF and COPD simultaneously occurred, the risk dramatically increased up to 3.73 (95\% confidence interval $=1.19-6.93, p<0.001$ ).

Conclusions Long-term follow-up showed higher mortality among elderly subjects affected by CHF or COPD. Simultaneous presence of CHF and COPD significantly increased the risk of death. Therefore, the presence of COPD in CHF patients should be considered a relevant factor in predicting high risk of mortality.

Keywords Chronic heart failure $\cdot$ Chronic obstructive pulmonary disease $\cdot$ Elderly $\cdot$ Mortality

\section{Introduction}

Chronic Obstructive Pulmonary Disease (COPD) is a very common disease being the fourth leading cause of death in western countries [1-3].

Epidemiologic data on COPD prevalence show a remarkable variability among cohorts due to differences in risk factor exposure and in data acquisition and analysis [4].

Nevertheless, it is well established that with increasing life expectancy and with the resulting prolonged exposure to risk factors, COPD incidence and prevalence are dramatically increasing in the general population and are expected to rise in the next decades [5-7].

While aging itself is considered a risk factor for COPD development, the aging process within the airways and the lung parenchyma mimics some of the structural changes 
associated with COPD [4]. It has been shown that COPDrelated morbidity and mortality increase with age and, more importantly, are significantly affected by comorbidities, representing a major social and economic burden for healthcare systems $[4,8]$.

Chronic Heart Failure (CHF) is highly prevalent with aging worldwide, reaching more than $20 \%$ prevalence in individuals aged $>80$ years and is becoming a significant health care issue in developed countries [7]. It represents the end stage in the cardiovascular disease (CVD) continuum, particularly of coronary heart disease and hypertension $[9,10]$. Despite continuous advances in the management of CHF patients, CHF morbidity and mortality remain high and are substantially affected by cardiac and non-cardiac comorbidity, especially in elderly patients [11, 12].

In the last few years, the coexistence of COPD and CHF is emerging as a challenge for healthcare professionals [13]. In fact, despite the lack of definitive data on the prevalence of the association between CHF and COPD, it is well known that smoking habit is a common risk factor for both diseases $[14,15]$.

These two chronic conditions share some pathophysiological mechanisms making the concurrent clinical diagnosis of COPD and CHF often difficult [13, 16]. Both COPD and $\mathrm{CHF}$ are characterized by low-grade systemic inflammation, which contributes to atherosclerosis progression, endothelial dysfunction, and myocardial damage [17, 18]. Moreover, as COPD progresses, pulmonary vascular bed is damaged leading to pulmonary circulation impairment and right heart failure with resultant detrimental effects on left ventricular function $[19,20]$.

It is well known that independently COPD and CHF strongly affect survival in elderly patients [21, 22], whereas very little data are available on mortality where patients are simultaneously affected by COPD and CHF. Thus, the aim of the present study was to assess whether the coexistence of COPD would affect long-term mortality in communitydwelling elderly subjects with CHF.

\section{Methods}

\section{Study population}

The "Osservatorio Geriatrico Regione Campania" was a cross-sectional study which started in 1992 in Campania, a Region in Southern Italy [23]. The study has been described elsewhere [24]. Briefly, the study enrolled 1780 subjects aged 65 and older which were randomly selected from the electoral rolls, resident in the five municipalities of Campania, and stratified by a three-step procedure according to age, sex, and size of urban unit [23, 24].
Of the 1780 enrolled subjects, $756(42.5 \%)$ male and 1024 (57.5\%) female; 448 subjects $(25.2 \%)$ refused to join in the study, resulting in a final study sample of 1332 subjects. Thus, the final overall participation rate was of $74.8 \%$. Mortality was assessed up to the end of 2003 by death certificates in 1297 of the 1332 (97.4\%) subjects enrolled in 1992; the remaining 35 (2.6\%) subjects were could not be reached. In $9(0.06 \%)$ of the 1297 enrolled subjects data on social support could not be collected [23, 24]. Thus the final study sample was of 1288 subjects ( 554 male and 734 female), among which 681 (52.9\%), 324 men (58.5\%), and 357 women (48.6\%) died [23, 24]. Study participants were reached at home or in their institution and carefully examined by physicians trained to administer a questionnaire encompassing cognitive and depression tests. Data concerning social status and demographic variables were also collected $[23,24]$. The "Osservatorio Geriatrico Campano" study was a single-center observational study performed by the University of University of Naples "Federico II"-Faculty of Medicine. For its characteristics by the time it was designed in 1992, the study underwent ethical evaluation by the institutional review board for formal approval but, since it was not required, it did not receive any approval number. All participants, however, signed an informed consent form.

The following variables were considered.

\section{Anthropometric measurement}

As part of the physical examination, height and weight were obtained with the participants in the standing position without shoes and heavy outer garments. Body Mass Index (BMI) was calculated as weight divided by height squared $\left(\mathrm{kg} / \mathrm{m}^{2}\right)[24]$.

\section{Blood pressure}

Blood pressure was measured according to the criteria of the Joint National Committee on Detection, Evaluation, and Treatment of High Blood Pressure [23]. Three blood pressure measurements at 2 min intervals were recorded when the subject had been sitting for approximately $1 \mathrm{~h}$, using a standard mercury sphygmomanometer [23]. The disappearance of sound (Korotkoff phase V) was used for the diastolic blood pressure reading. The mean value of the last two measurements was used for the analysis. Systolic, diastolic, and pulse (systolic-diastolic) blood pressure were recorded [23].

\section{CHF diagnosis}

Chronic Heart Failure diagnosis was considered when participants reported they were previously diagnosed 
with $\mathrm{CHF}$, and/or they received specific treatment with diuretics, digitalis, and/or vasodilators [25]. The diagnosis was confirmed through the physical examination and a careful review of participant's medical records to verify the presence of atrial fibrillation, cardiomegaly, and pulmonary edema on chest X-ray, and/or evidence of left ventricular dilation and/or global or segmental wall motion abnormalities. Moreover, the following signs and symptoms were assessed and characterized: dyspnea, orthopnea, tachycardia, jugular venous distension, abdomino-jugular reflux, pulmonary rales, third sound, and edema [25]. When physical examination was performed, these signs and symptoms were not categorized into major and minor. All subjects with chronic heart failure were identified and characterized at the enrollment in 1992 and "de novo" heart failure cases were not identified after 1992 [25].

\section{COPD diagnosis}

The diagnosis of COPD was considered possible when participants reported previous clinical diagnosis of COPD, and/or they received specific treatment with inhaled beta-agonist and steroids either alone or in combination [5]. The diagnosis was confirmed through the physical examination and a careful review of participant's medical records of pulmonary function test (PFT) records. In particular, subjects were considered as having COPD if they had values of FEV1/FVC $<0.70$ and FEV1 from $\geq 80 \%<50 \%$ predicted at PFT [5].

COPD diagnosis was confirmed with history of exposure to risk factors, presence of symptoms (dyspnea chronic cough and expectoration), and obstructive ventilator pattern, in terms of Tiffeneau Index (FEV1/ $\mathrm{FVC}<0.70 \%)$, FEV1, and FVC at spirometry [5].

\section{Chronic conditions, cognitive function, and depression}

Chronic conditions were evaluated from the past medical history analysis and confirmed by a trained physician through a careful clinical examination. Comorbidity was evaluated using the Cumulative Illness Rating Scale Severity Index and Charlson Comorbidity Index [8]. The Italian version of the Mini-Mental State Examination (MMSE) validated by Measso [23] was used to evaluate participant's cognitive mental status. Cognitive impairment was defined as an MMSE score of less than 24 [23]. The Geriatric Depression Scale (GDS) [26] was used to evaluate depressive symptoms. Data drug consumption (class and administration) were collected.

\section{Disability}

Disability was assessed the Basic Activities of Daily Living (BADL) [27] score exploring the following domains: bathing or showering, dressing, eating, getting in and out of bed, transferring from bed to chair and using the toilet [27]. For each item a four-point score was attributed according to the function impairment: "uses no help to perform the activity", "uses a device to perform the activity", "uses assistance of another person to perform the activity", "does not perform the activity". Subjects who could not perform the function without help were considered disabled.

\section{Social support}

Social support analysis assessed three categories commonly described as social networks, social relationships, and economic supports. Social networks refer to everyday contacts including size, density, reciprocity, durability, intensity, and frequency of contacts. Social relationships refer to the existence, quantity, and type of relationships [28]. The final score of these items ranges from 0 to 17 , the lowest social support level corresponding to the highest score. The score was divided in tertiles: 0 to 5 for the highest level of social support (score $=1$ ), 6 to 12 for medium (score $=2$ ), and 13 to 17 for lowest social support (score $=3$ ).

\section{Statistical analysis}

Data were collected and analyzed using the SPSS 13.0 statistics package. Baseline characteristics of the sample are expressed as mean \pm standard deviation. Study participants were stratified based on the presence and absence of CHF, on the presence and absence of COPD, and on the presence and absence of both CHF and COPD. Differences in sex (female), current smoking, former smoking, MMSE $<24$, and BADL ( $\geq 1$ function lost) were assessed with $\chi^{2}$ analysis. Differences in age, BMI, heart rate, systolic, diastolic, and pulse blood pressure, Cumulative Illness Rating Scale (CIRS), Charlson Comorbidity Index, drugs number, GDS, and social support were evaluated by one-way ANOVA. To evaluate the effects of CHF, COPD and of the coexistence of $\mathrm{CHF}$ and COPD on long-term mortality multivariate analysis was performed independently of the role exerted by age, sex female, BMI, heart rate, pulse pressure, NYHA class, CIRS, drugs number, current smoking, former smoking, MMSE $<24$, GDS, BADL $(\geq 1$ lost), and social support. Hazard ratio (HR) and 95\% confidence interval (CI) were calculated for each variable. Since interaction between $\mathrm{CHF}$ and COPD $(\mathrm{HR}=3.41,95 \% \mathrm{CI}=1.92-6.12)$, between $\mathrm{CHF}$ and $\mathrm{CHF}$ and COPD together $(\mathrm{HR}=3.57$, 95\% CI $=1.93-6.42,<0.001$ ), and between COPD by coexistence of $\mathrm{CHF}$ and COPD (HR $=1.47$, CI 95\% 1.18-1.85, 
$p<0.001)$ were significant, separate multivariate analyses on the role of CHF, COPD and coexistence of CHF and COPD were performed. Finally, survival curves with Cox regression analysis were performed by selecting the mortality of all subjects, subjects with CHF or COPD, and in subjects with coexistence of CHF and COPD. A $p<0.05$ was considered significant.

\section{Results}

Subjects with CHF were older than subjects without CHF. In addition, heart rate, NYHA III-IV class, Charlson comorbidity Index, drugs number, MMSE $<24$, GDS, BADL lost $\geq 1$, low social support were higher in CHF subjects (Table 1).

Subjects with COPD were older than subjects without COPD. As expected, male sex, current, and former smoking status was more prevalent in COPD subjects. Heart rate, NYHA III-IV class, and drugs number were higher in elderly subjects with COPD than in those without. Moreover, Charlson comorbidity Index, GDS score, and the prevalence of BADL lost $\geq 1$ were higher in COPD subjects (Table 1).

Subjects with coexisting CHF and COPD were older and former smokers were more prevalent. In addition, in these subjects heart rate, pulse BP, NYHA III-IV class, Charlson comorbidity Index, drugs number, MMSE $<24$, GDS, BADL lost $\geq 1$, and low social support were higher (Table 1). Interestingly, when compared subjects affected by isolated CHF or COPD, all these clinical characteristics were significantly more compromised in subjects with coexisting CHF and COPD (Table 1).

Figure 1 shows mortality, at 12 years follow-up, in all subjects, in subjects with and without CHF, in subject with or without COPD, and in subjects with and without coexisting CHF and COPD. In all subjects mortality was $46.7 \%$. Mortality was $68.6 \%$ in the presence and $31.4 \%$ in the absence of CHF $(p<0.001)$ while it was the $56.9 \%$ in the presence and $43.1 \%$ in the absence of COPD. Mortality was $13.8 \%$ in the absence and $86.2 \%$ in the presence of both CHF and COPD $(p<0.001)$, being significantly higher than in CHF or COPD alone (Fig. 1).

Both CHF $(\mathrm{HR}=1.67,95 \%$ CI $1.15-3.27, p<0.031)$ and $\mathrm{COPD}(\mathrm{HR}=1.27,95 \% \mathrm{CI}=1.08-1.85, p<0.042)$ were predictive of long-term mortality in a multivariate model adjusted for sex, age, and several other variables (Table 2). When multivariate analysis was performed to assess the role of coexisting CHF and COPD on the risk of long-term mortality, HR dramatically increased up to 3.73 (95\% CI $=1.19-6.93, p<0.001)$ (Table 2). Figure 2 represents survival curves of Cox regression analysis performed by selecting the mortality of all subjects, subject with $\mathrm{CHF}$ or COPD, and of subjects with both CHF and COPD. It is worth of note that survival curve of $\mathrm{CHF}$ and COPD subjects was more steep with respect to those of individuals with CHF or COPD alone.

\section{Discussion}

In our 12 year follow-up study, elderly subjects with CHF showed a mortality of $\approx 70 \%$, those with COPD $\approx 60 \%$ while in presence of CHF and COPD, long-term mortality was $\approx 90 \%$.

More importantly, multivariate analysis indicates that the risk of mortality was nearly tripled in elderly subjects with both $\mathrm{CHF}$ and COPD when compared to CHF or COPD alone.

This dramatic impact on long-term survival highlights the relevance of coexistence of CHF and COPD in the elderly population.

\section{COPD and mortality in the elderly}

COPD is becoming a leading cause of morbidity and mortality worldwide resulting in a substantial increase in economic and social burden [1-4]. A comprehensive international population-based survey aiming to address COPD prevalence and risk factors in the general adult population across countries by age, sex, and smoking status reported a prevalence of COPD among subjects older than 70 years old rising to more than 20\% [29]. In our sample, we observed a prevalence of COPD subjects of $31.1 \%$ with a higher mean age and male prevalence compared to subjects without COPD. As expected, former and current smoker rates were higher in subjects with COPD than those without. In our sample, COPD subjects exhibited high presence of comorbidity, were frequently in polypharmacy, and had higher disability rates with respect to subjects without COPD. COPD subjects had higher heart rates and were reported to be more frequently in NYHA class III-IV, which further demonstrates difficulties in differential diagnosis between COPD and CHF due to similar clinical manifestations and symptoms $[13,16]$. However, in our study, in addition to physical examination, CHF and COPD diagnoses were confirmed by a medical record review.

Finally, COPD-related mortality is reported to rise with age and, therefore, is expected to increase with increased life expectancy [4]. In our sample, we report a 12 year mortality of almost $60 \%$ in subjects with COPD compared to those without. Accordingly, in our sample Cox regression analysis showed that COPD subjects were associated with a significantly increased risk of death (HR 1.27). 


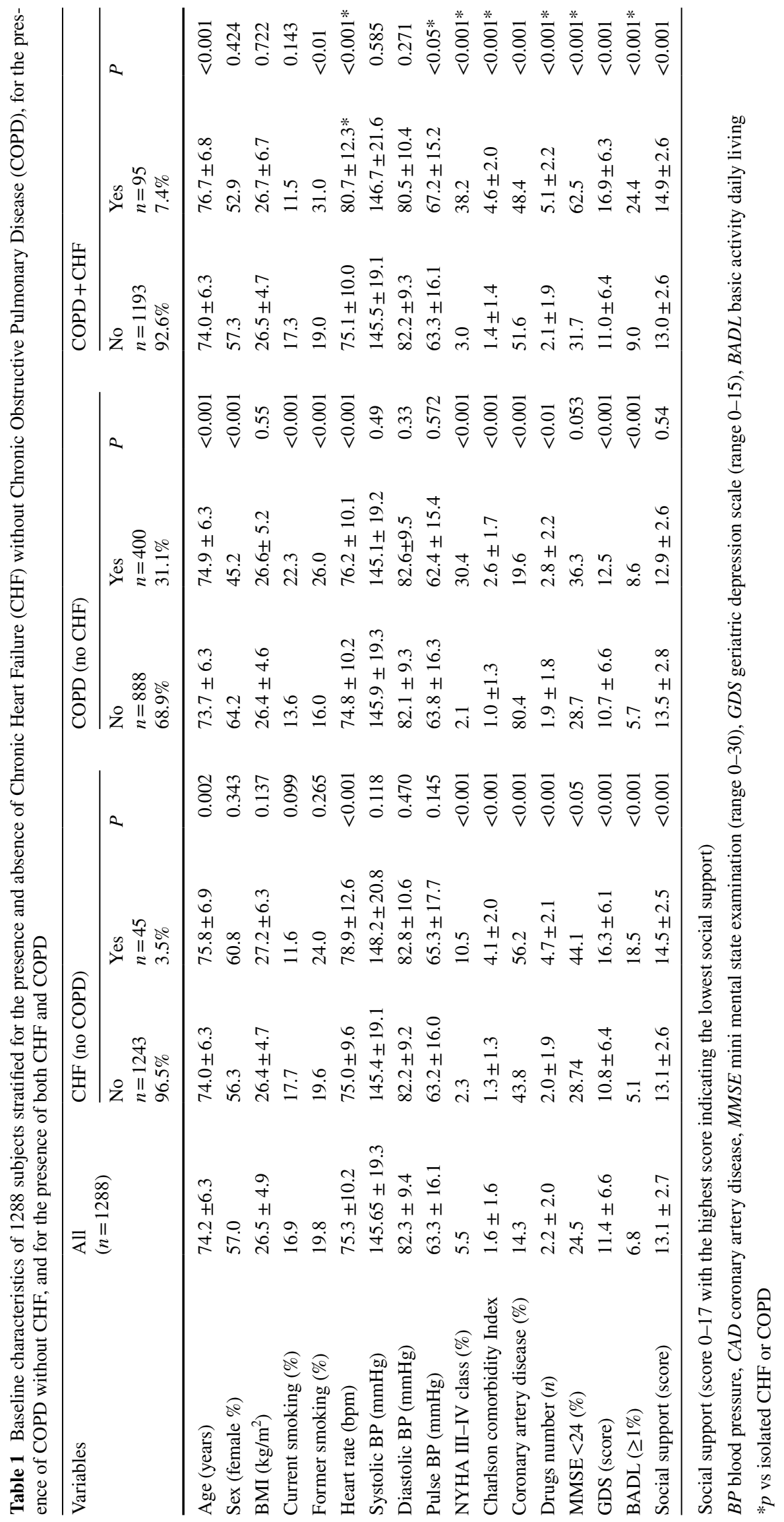




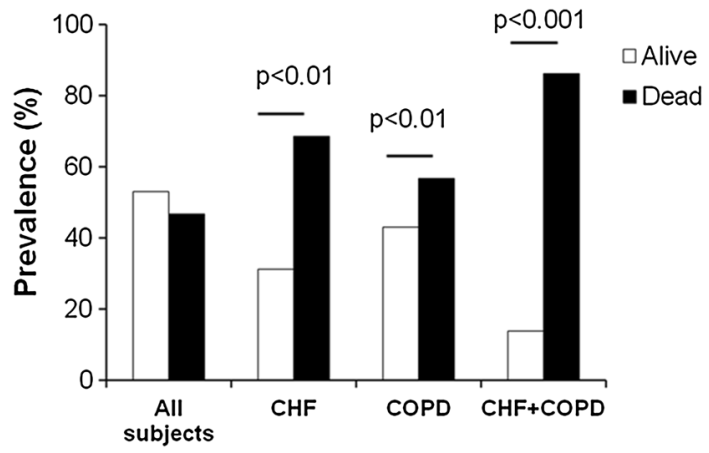

Fig. 1 Prevalence of long-term mortality (12 years follow-up) in all subjects, in subjects with chronic heart failure (CHF) without chronic obstructive pulmonary disease (COPD), with COPD without CHF, and with both CHF and COPD

\section{CHF and mortality in the elderly}

CHF is highly prevalent in aging populations worldwide in individuals aged $>80$ years [12]. CHF is considered a healthcare issue in developed countries [9] and is often associated with relevant cardiac and non-cardiac comorbidities leading to several clinical characteristics common to geriatric patients (comorbidity, polypharmacy, disability). Thus, despite continuous advances in therapy, CHF morbidity and mortality remain high [12]. In accordance with the fact that CHF is often present in subjects with multiple cardiac and non-cardiac comorbidities [8], in our sample the prevalence of isolated CHF was $3.5 \%$. These subjects were older than subjects without $\mathrm{CHF}$ and, as expected, had higher heart rate and NYHA class III-IV. Interestingly, subjects with isolated CHF had higher comorbidity, more prescription drugs and were more cognitively impaired, disabled, and depressed. In CHF subjects, mortality rates were more than doubled when compared to subjects without CHF (68.6 vs $31.4 \%$ ) with a related high predictive power of CHF on mortality (HR 1.67).

\section{CHF in the presence of COPD and mortality in the elderly}

Despite the lack of definitive data on the prevalence of the association of CHF and COPD, the coexistence of these two chronic conditions is very common and is becoming a challenge for healthcare professionals [13]. In recent years, several studies have focused on the impact of COPD and CHF on clinical outcomes both in the acute and chronic setting, as therapeutic cornerstones of these two conditions may significantly worsen each other [16]. In our sample, subjects with coexisting COPD and CHF were $7.4 \%$. These subjects had a higher mean age and prevalence of female sex, higher comorbidities, and more prescription drugs with respect to subjects affected by COPD or CHF alone. Our prevalence data differ from those reported in other studies $[15,16,21,22]$ confirming the observation

Table 2 Cox regression analysis on 12-years mortality

\begin{tabular}{|c|c|c|c|c|c|c|c|c|c|}
\hline \multirow[t]{2}{*}{ Variables } & \multicolumn{3}{|c|}{ CHF (no COPD) } & \multicolumn{3}{|c|}{ COPD (no CHF) } & \multicolumn{3}{|c|}{$\mathrm{COPD}+\mathrm{CHF}$} \\
\hline & HR & CI $95 \%$ & $P$ & HR & CI $95 \%$ & $P$ & HR & CI $95 \%$ & $P$ \\
\hline Age & 1.15 & $1.12-1.18$ & $<0.001$ & 1.15 & $1.11-1.18$ & 0.000 & 1.15 & $1.11-1.18$ & 0.000 \\
\hline Sex female & 0.40 & $0.27-0.58$ & $<0.001$ & 0.40 & $0.27-0.59$ & 0.000 & 0.40 & $0.27-0.58$ & 0.000 \\
\hline BMI & 0.98 & $0.95-1.01$ & 0.279 & 0.98 & $0.95-1.01$ & 0.259 & 0.98 & $0.95-1.01$ & 0.304 \\
\hline Former smoking & 1.13 & $0.72-1.76$ & 0.575 & 1.12 & $0.72-1.74$ & 0.608 & 1.12 & $0.72-1.75$ & 0.598 \\
\hline Current smoking & 1.09 & $0.70-1.72$ & 0.683 & 1.08 & $0.69-1.70$ & 0.716 & 1.09 & $0.70-1.72$ & 0.682 \\
\hline Heart rate (bpm) & 0.99 & $0.98-1.01$ & 0.810 & 0.99 & $0.98-1.01$ & 0.872 & 0.99 & $0.98-1.01$ & 0.825 \\
\hline Pulse BP (mmHg) & 1.00 & $0.99-1.01$ & 0.331 & 1.00 & $0.99-1.01$ & 0.365 & 1.00 & $0.99-1.01$ & 0.303 \\
\hline NYHA & 2.34 & $1.16-4.71$ & 0.016 & 2.61 & $1.32-5.13$ & 0.005 & 3.49 & $1.84-6.61$ & 0.000 \\
\hline Charlson comorbidity index & 1.10 & $1.01-1.14$ & $<0.05$ & 1.01 & $0.87-1.17$ & 0.846 & 1.15 & $1.07-1.20$ & $<0.05$ \\
\hline Drugs number $(n)$ & 1.13 & $1.05-1.23$ & 0.001 & 1.15 & $1.06-1.24$ & 0.001 & 1.20 & $1.06-1.38$ & 0.001 \\
\hline MMSE <24 (\%) & 1.63 & $1.21-2.19$ & 0.001 & 1.64 & $1.23-2.19$ & 0.001 & 1.72 & $1.29-2.29$ & 0.038 \\
\hline GDS (score) & 1.00 & $0.97-1.02$ & 0.779 & 1.00 & $0.97-1.02$ & 0.786 & 1.00 & $0.97-1.02$ & 0.759 \\
\hline $\operatorname{BADL}(\geq 1 \%)$ & 2.03 & $1.00-4.12$ & $<0.05$ & 2.02 & $1.01-4.15$ & $<0.05$ & 2.24 & $1.04-4.45$ & 0.032 \\
\hline Social support (score) & 1.63 & $1.28-2.09$ & 0.000 & 1.62 & $1.27-2.08$ & 0.000 & 1.78 & $1.29-2.18$ & 0.000 \\
\hline $\mathrm{CHF}$ & 1.67 & $1.15-3.27$ & 0.031 & - & - & - & - & - & - \\
\hline COPD & - & - & - & 1.27 & $1.08-1.85$ & 0.042 & - & - & - \\
\hline $\mathrm{CHF}+\mathrm{COPD}$ & - & - & - & & & & 3.73 & $1.19-6.93$ & $<0.001$ \\
\hline
\end{tabular}

$H R$ hazard ratio, $C I$ confidence interval, $C H F$ chronic hear failure, $C O P D$ chronic obstructive pulmonary disease, $B P$ blood pressure, $M M S E$ mini mental state examination, $G D S$ geriatric depression scale; $B A D L$ basic activity daily living 


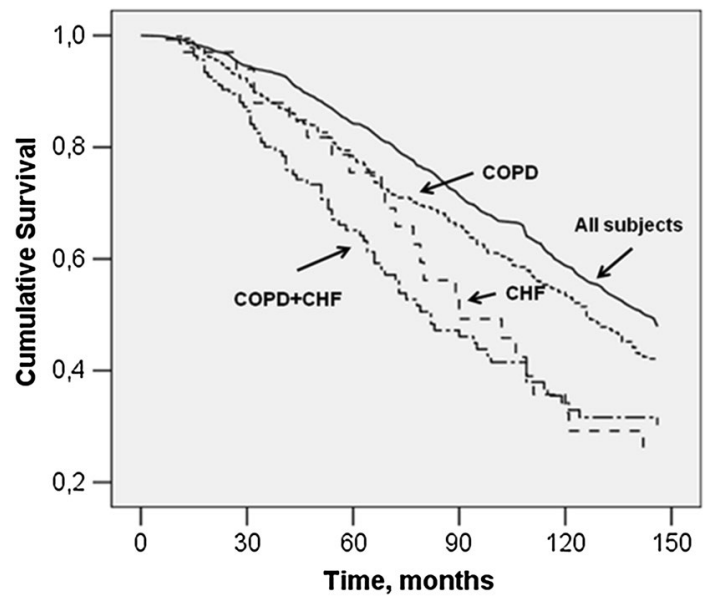

Fig. 2 Cox regression adjusted survival curve in all subjects, in subjects with Chronic Heart Failure (CHF) without Chronic Obstructive Pulmonary Disease (COPD), with COPD without CHF, and with both CHF and COPD

that COPD diagnosis remains underestimated in the general population, especially in subjects with mild symptoms [30].

The coexistence of COPD and CHF often raises diagnostic issues as the two conditions share some pathophysiological mechanisms $[13,16]$, which can also be responsible for poorer prognosis. In fact, it is well known that smoking is the key risk factor for COPD which, in turn, is associated with a 50\% increased risk of developing CHF [14, 15]. In addition, both COPD and CHF are characterized by lowgrade systemic inflammation, which is an established determinant of atherosclerosis progression, endothelial dysfunction, and myocardial damage $[14,15]$. As COPD worsens, the pulmonary vascular bed becomes involved leading to increased pulmonary vascular resistance, pulmonary hypertension, and right heart failure which ultimately affects left ventricular function in a vicious circle [19, 20]. In our sample, former, but not current, smokers were more prevalent in subjects with coexisting COPD and CHF with respect to those with COPD or CHF alone. In addition, subjects with COPD and CHF had a significant increase of pulse pressure values and heart rate compared to subjects with either COPD or CHF alone. This observation may reflect a reduced peripheral compliance probably due to both increased aortic stiffness and reduced thoracic compliance [31]. Interestingly, we observed a higher prevalence of subjects with coexisting COPD and CHF in NYHA functional class III-IV (38\%). All these observations support the idea that COPD and CHF progression significantly affect each other's clinical status and outcomes [13-17].

It is well known that the isolated presence of either COPD or CHF is characterized by high disability and low survival in elderly patients $[21,22]$. Our data clearly show a steep increase in loss of ADL and, more importantly, in mortality, at 12 year follow-up, in subjects with coexisting COPD and CHF with respect to subjects with CHF or COPD alone and a strong predictive role for the coexistence of COPD and CHF on mortality. In fact, both CHF $(\mathrm{HR}=1.67)$ and $\mathrm{COPD}(\mathrm{HR}=1.27)$ were predictive of long-term mortality in a multivariate model. When multivariate analysis was performed to evaluate the role of coexisting CHF and COPD on the risk of long-term mortality, HR dramatically increased up to 3.73 .

More interestingly, we observed a remarkable increase of mortality in subjects with coexisting COPD and CHF between baseline and 90 months. This observation could be due to the impact of COPD on the acceleration of cardiovascular disease progression leading to $\mathrm{CHF}$ and viceversa. Recently, coronary artery disease has been considered a possible factor that potentiates COPD morbidity and mortality, especially in elderly subjects, probably due to low-grade systemic inflammation hypothesis which links CAD, COPD, and aging [32]. In our sample, the prevalence of CAD in subjects with COPD and CHF was $48.4 \%$ $(n=46)$. From baseline to 90 months of follow-up, mortality rates among these subjects were close to $90 \%(86.9 \%$, $n=40$ ) highlighting the relevance of cardiovascular disease in CHF and COPD subjects.

\section{Limitations of the Study}

The results of this study should be evaluated taking account of several limitations. The first limitation is that of being retrospective, thus missing incident cases. Moreover, due to the recruitment of community-dwelling subjects, the study was not designed to collect data on hospitalizations. Finally, left and right ventricular function data were not available for all subjects, and thus could not be considered in the analysis.

\section{Conclusions}

Our study indicates that CHF-related mortality at 12 years follow-up is higher in subjects with COPD compared to those without. Thus, the presence of COPD may be considered a new prognostic factor to identify CHF subjects at high risk of mortality, especially when CHF is due to coronary artery disease. There is need to carefully evaluate the early occurrence of comorbidities in both CVD and COPD patients to appropriately treat the patients and delay the synergistic pathophysiological progression of both diseases.

Further investigations are required to better identify mechanisms underlying the detrimental effects observed which may lead to better management of this growing subset of elderly patients. 


\section{Compliance with ethical standards}

Conflict of interest On behalf of all authors, the corresponding author states that there is no conflict of interest.

Ethical approval All procedures performed in studies involving human participants were in accordance with the ethical standards of the institutional and/or national research committee and with the 1964 Helsinki declaration and its later amendments or comparable ethical standards.

Informed consent Informed consent was obtained from all individual participants included in the study.

\section{References}

1. US Burden of Disease Collaborators (2013) The state of US health,1990-2010: burden of diseases, injuries, and risk factors. JAMA 310:591-608

2. de Laurentiis G, Paris D, Melck D et al (2013) Separating smoking-related diseases using NMR-based metabolomics of exhaled breath condensate. J Proteome Res 12:1502-1511

3. Esposito V, Lucariello A, Savarese L et al (2012) Morphology changes in human lung epithelial cells after exposure to diesel exhaust submicron particles (PM(1.0)) and pollen allergens. Environ Pollut 171:162-167

4. From the global strategy for the diagnosis, management and prevention of COPD, Global Initiative for Chronic Obstructive Lung Disease (GOLD) 2015. http://www.goldcopd.org/.

5. Galizia G, Cacciatore F, Testa G, Della-Morte D et al (2011) Role of clinical frailty on long-term mortality of elderly subjects with and without chronic obstructive pulmonary disease. Aging Clin Exp Res 23:118-125

6. Battaglia S, Basile M, Scichilone N, Bellia V (2015) Prevalence of Co-morbidities and Severity of COPD. COPD 12:390-394

7. Sansores RH, Ramirez-Venegas A, Hernandez-Zenteno R, Mayar-Maya ME, Perez-Bautista OG, Velazquez UM (2013) Prevalence and diagnosis of chronic obstructive pulmonary disease among smokers at risk. A comparative study of case-finding vs screening strategies. Respir Med 107:580-586

8. Testa G, Cacciatore F, Galizia G et al (2009) Charlson Comorbidity Index does not predict long-term mortality in elderly subjects with chronic heart failure. Age Ageing 38:734-740

9. Abete P, Testa G, Della-Morte D et al (2013) Treatment for chronic heart failure in the elderly: current practice and problems. Heart Fail Rev 18:529-551

10. Ghali JK, Massie BM, Mann DL, Rich MW (2010) Heart failure guidelines, performance measures, and the practice of medicine: mind the gap. J Am Coll Cardiol 56:2077-2080

11. Testa G, Della-Morte D, Cacciatore F et al (2013) Precipitating factors in younger and older adults with decompensated chronic heart failure: are they different? J Am Geriatr Soc 61:1827-1828

12. Vader JM, Rich MW (2015) Team-based care for managing noncardiac conditions in patients with heart failure. Heart Fail Clin 11:419-429

13. Ishitzu T (2014) Chronic obstructive pulmonary disease: Pathophysiological impact on heart failure in real clinical situation. $\mathrm{J}$ Cardiol 64:250-252

14. Sin DD, Man SF (2003) Why are patients with chronic obstructive pulmonary disease at increased risk of cardiovascular diseases? The potential role of systemic inflammation in chronic obstructive pulmonary disease. Circulation 107:1514-1519
15. Sin DD, Wu L, Man SF (2005) The relationship between reduced lung function and cardiovascular mortality: a population-based study and a systematic review of the literature. Chest 127:1952-1959

16. Mentz RJ, Fiuzat M, Wojdyla DM et al (2012) Clinical characteristics and outcomes of hospitalized heart failure patients with systolic dysfunction and chronic obstructive pulmonary disease: findings from OPTIMIZE-HF. Eur J Heart Fail 14:395-403

17. Georgiopoulou VV, Kalogeropoulos AP, Psaty BM et al (2011) Lung function and risk for heart failure among older adults: the Health ABC Study. Am J Med 124:334-341

18. Bianco A, Mazzarella G, Turchiarelli V et al (2013) Adiponectin: an attractive marker for metabolic disorders in chronic obstructive pulmonary disease (COPD). Nutrients 5:4115-4125

19. Haddad F, Doyle R, Murphy DJ, Hunt SA (2008) Right ventricular function in cardiovascular disease, part II: pathophysiology, clinical importance, and management of right ventricular failure. Circulation 117:1717-1731

20. Louie EK, Rich S, Levitsky S, Brundage BH (1992) Doppler echocardiographic demonstration of the differential effects of right ventricular pressure and volume overload on left ventricular geometry and filling. J Am Coll Cardiol 19:84-90

21. Scott DA, Woods B, Thompson JC et al (2015) Mortality and drug therapy in patients with chronic obstructive pulmonary disease: a network meta-analysis. BMC Pulm Med 15:145-157

22. Martínez-Sellés M, Doughty RN, Poppe K et al (2012) MetaAnalysis Global Group In Chronic Heart Failure (MAGGIC). Gender and survival in patients with heart failure: interactions with diabetes and aetiology. Results from the MAGGIC individual patient meta-analysis. Eur J Heart Fail 14:473-479

23. Cacciatore F, Abete P, de Santis D, Longobardi G, Ferrara N, Rengo F (2005) Mortality and blood pressure in elderly people with and without cognitive impairment. Gerontology 51:53-61

24. Cacciatore F, Gallo C, Ferrara N et al (2008) Morbidity patterns in aged population in southern Italy. A survey sampling. Arch Gerontol Geriatr 263:201-213

25. Testa G, Cacciatore F, Galizia G et al (2010) Waist circumference but not body mass index predicts long-term mortality in elderly subjects with chronic heart failure. J Am Geriatr Soc 58:1433-1440

26. Testa G, Cacciatore F, Galizia G et al (2011) Depressive symptoms predict mortality in elderly subjects with chronic heart failure. Eur J Clin Invest 41:1310-1317

27. Katz S, Ford AB, Moskowitz RW, Jackson BA, Jaffe MW (1963) Studies of illness in the aged. The index of BADL; a standardized measure of biological and psycological functions. JAMA 185:94-99

28. Cacciatore F, Testa G, Galizia G et al (2013) Clinical frailty and long-term mortality in elderly subjects with diabetes. Acta Diabetol 50:251-260

29. Buist AS, McBurnie MA, Vollmer WM et al (2007) International variation in the prevalence of COPD (the BOLD Study): a population-based prevalence study. The Lancet 370:741-750

30. Martinez CH, Mannino DM, Jaimes FA et al (2015) Undiagnosed obstructive lung disease in the US: associated factors and long-term mortality. Ann Am Thorac Soc 12:1788-1795. doi:10.1513/AnnalsATS.201506-3880C

31. Weir-McCall JR, Struthers AD, Lipworth BJ, Houston JG (2015) The role of pulmonary arterial stiffness in COPD. Respir Med 109:1381-1390

32. Roversi S, Roversi P, Spadafora G, Rossi R, Fabbri LM (2014) Coronary artery disease concomitant with chronic obstructive pulmonary disease. Eur J Clin Invest 44:93-102 